\title{
Interrupción de vena cava inferior mediante filtros de inserción percutánea: Indicaciones y resultados en 287 pacientes
}

\author{
Ivette Arriagada J, Renato Mertens M, Francisco Valdés E, \\ Albrecht Krämer Sch, Leopoldo Mariné M, \\ Michel Bergoeing R, Sebastián Soto G, \\ Jeannette Vergara $\mathbf{G}$ a, Magaly Valdebenito $\mathbf{G}$ a.
}

\section{Percutaneous inferior vena cava filters: Indications and results in 287 patients}

Background: Anticoagulation is the treatment of choice for deep vein thrombosis (DVT) and pulmonary embolism (PE). Occasionally this treatment is contraindicated or fails to prevent PE. In these patients, inferior vena caval (IVC) interruption is indicated and insertion of a filter is the most commonly performed procedure. Aim: To report the experience with IVC filters. Material and methods: Retrospective review of all medical records and operative protocols of patients subjected to IVC filter implantations. Follow up was performed by telephone contact with the patient, relatives or primary physicians, ambulatory consultation or by death certificates. Results: During the period 1993-2005 we implanted IVC filters on 287 patients, $55.4 \%$ male, average age: 62.1 yrs (17-99). Indications for the procedure were DVT or PE and contraindication of anticoagulation in 141 patients (49.1\%), DVT or PE and complication of anticoagulation in 65 patients (22.6\%), prophylaxis in 39 patients (13.6\%), massive PE or poor respiratory function in 31 patients (10.8\%), paradoxal emboli in 4 patients (1.4\%) and other causes in seven patients. All percutaneous devices were successfully inserted. There was no morbidity or mortality related to the procedure. The most frequent access site was the internal jugular vein (66.6\%). In 24 patients (8.4\%) the filter was intentionally deployed above the renal veins. Six patients (2.1\%) were lost to follow up after discharge. A mean follow up of 41.5 months was achieved. Ninety one patients died, with a 5 years survival of $64.7 \%$. Symptomatic recurrent PE occurred in 6 patients $(2.1 \%)$ and was the cause of death on 3 of them (1\%), DVT has been detected in 22 patients (7.7\%) during the follow up period. Conclusions: IVC filter implantation is a safe and effective short and long term measure to prevent PE and its consequences (Rev Méd Chile 2007; 135: 351-8).

(Key words: Deep vein thrombosis; Filters, inferior vena cava; Thromboembolism)

Recibido el 28 de junio, 2006. Aceptado el 11 de septiembre, 2006.

Cirugía Vascular y Endovascular, Departamento de Enfermedades Cardiovasculares y División de Cirugía, Pontificia Universidad Católica de Chile. Santiago de Chile. aEnfermera Universitaria

Correspondencia a: Dr. Renato Mertens M. Dirección: Apoquindo 3990 oficina 601, Santiago, Chile. Fax: (56 2) 6326812. E mail: rmertens@med.puc.cl 
$\mathrm{E}^{1}$ tratamiento de la trombosis venosa profunda de extremidades inferiores y de la embolia pulmonar, es la anticoagulación. Sin embargo, no siempre constituye una opción terapéutica, dado que puede estar contraindicada por riesgo de sangrado, por presentar complicaciones hemorrágicas o fracasar en prevenir la progresión de la enfermedad tromboembólica o la embolia pulmonar. La embolia pulmonar es una importante causa de morbimortalidad, potencialmente evitable, en el paciente hospitalizado.

En las situaciones descritas, el procedimiento de elección es el implante de un filtro en la vena cava inferior, con el fin de impedir el paso de émbolos desde las extremidades inferiores hacia la circulación pulmonar.

En 1983 iniciamos en nuestro centro el uso de filtros en vena cava inferior, utilizando dispositivos que requerían de denudación para su inserción ${ }^{1}$. El objetivo de esta comunicación es revisar nuestras indicaciones y resultados a corto y largo plazo, utilizando los dispositivos de inserción percutánea disponibles en la actualidad.

\section{MATERIAL Y MÉTODOS}

Se incluyen en este estudio todos los pacientes a quienes les fue indicado e instalado un filtro de vena cava inferior de inserción percutánea, por los miembros de la sección de Cirugía Vascular y Endovascular del Hospital Clínico de la Pontificia Universidad Católica de Chile.

Se revisaron retrospectivamente los registros y protocolos operatorios, evaluando características demográficas, indicaciones y detalles técnicos del procedimiento. El seguimiento se realizó mediante contacto telefónico con el paciente, familiares 0 médico tratante, consulta ambulatoria u obtención de certificados de defunción emitidos por el Servicio de Registro Civil e Identificación. Se consideró como seguimiento completo a aquellos pacientes que fueron controlados en forma ambulatoria o contactados telefónicamente al momento del cierre del estudio y como seguimiento parcial a aquellos que, habiendo conseguido seguimiento por un periodo variable, no fueron contactados al momento del cierre del estudio y sólo se pudo determinar su condición de vivo o muerto, mediante certificados de defunción.
Técnica utilizada. Los filtros fueron insertados en sala de operaciones por un cirujano vascular, bajo anestesia local, utilizando técnica aséptica y acceso percutáneo con técnica de Seldinger. Se mantuvo monitorización de la hemodinamia e intercambio gaseoso. En ocasiones, según la gravedad del paciente, se contó con la colaboración de un anestesiólogo. En aquellos pacientes en quienes la anticoagulación no estaba contraindicada, ésta se mantuvo durante y después del procedimiento. Se utilizó radioscopia para controlar el avance, localización y despliegue del dispositivo, generada mediante un angiógrafo portátil $\left(\right.$ Siremobil $^{\circledR}$, Siemens ${ }^{\circledR}$, Alemania u OEC $9800^{\circledR}$, General Electric $^{\circledR}$, Estados Unidos de Norteamérica).

Durante el procedimiento, se evaluó la anatomía venosa y eventual presencia de trombos en la vena cava inferior mediante ilio-cavografía con medio de contraste no iónico, inmediatamente previa al despliegue del filtro.

La vía de inserción fue la vena yugular interna, femoral común o subclavia, dependiendo de las condiciones anatómicas y la preferencia del operador.

La hemostasia del sitio de punción se obtuvo mediante compresión directa. Luego del procedimiento, el paciente fue trasladado a su unidad de origen.

\section{RESULTADOS}

Entre enero de 1993 y septiembre de 2005 se indicó e implantó un filtro de vena cava a 287 pacientes, previo consentimiento informado. El promedio de edad fue 62,1 años (rango 17-99). El 55,4\% ( $\mathrm{n}=159$ ) eran hombres. En la Tabla 1 se enumeran las comorbilidades presentes al momento del evento tromboembólico. Las más frecuentes fueron enfermedades cardiovasculares, presentes en 52,6\%, seguidas por enfermedades neoplásicas, que afectaban a $28,9 \%$ de los pacientes.

En 194 pacientes $(67,6 \%)$ se confirmó el diagnóstico de trombosis venosa profunda. De éstos, 33 tenían trombosis que se extendía hasta la vena cava inferior. Un paciente se presentó con flegmasia cerulea dolens y requirió de trombectomía venosa simultánea. A 164 pacientes $(57,1 \%)$ se les diagnosticó embolia pulmonar. Todos tuvieron confirmación con imágenes: tomografía axial com- 
Tabla 1. Comorbilidad al momento del evento tromboembólico venoso en 287 pacientes tratados con filtros de vena cava inferior

\begin{tabular}{|lrl|}
\hline Patología & Número de pacientes (\%) \\
\hline Enfermedades cardiovasculares & 151 & $(52,6)$ \\
Neoplasias & 81 & $(28,9)$ \\
Enfermedades endocrinas, nutricionales, metabólicas & 66 & $(23)$ \\
Enfermedades del sistema nervioso y órganos de los sentidos & 55 & $(19,2)$ \\
Enfermedades psiquiátricas & 47 & $(16,4)$ \\
Enfermedades del sistema respiratorio & 34 & $(11,8)$ \\
Enfermedades del sistema digestivo & 40 & $(13,9)$ \\
Enfermedades del sistema genitourinario & 28 & $(9,8)$ \\
Enfermedades infecciosas & 12 & $(4,2)$ \\
Enfermedades del sistema musculoesquelético y tejido conjuntivo & 7 & $(2,4)$ \\
\hline
\end{tabular}

putarizada, cintigrama ventilación-perfusión o angiografía pulmonar.

Las indicaciones para interrupción de vena cava inferior se detallan en la Tabla 2. La más frecuente fue contraindicación de tratamiento anticoagulante $(49,1 \%)$, seguida de complicación del tratamiento anticoagulante, por hemorragia o fracaso del tratamiento $(22,6 \%)$. La Tabla 3 enumera las causas que contraindicaron o complicaron el tratamiento anticoagulante.

Los implantes de filtros profilácticos se realizaron, en su mayoría, en presencia de enfermedad

\section{Tabla 2. Indicación de interrupción de la vena cava inferior en 287 pacientes (TAC =tratamiento anticoagulante, TEP =tromboembolismo pulmonar)}

\begin{tabular}{|lrl|}
\hline Indicación & Pacientes (\%) \\
\hline Contraindicación de TAC & $141(49,1)$ \\
Complicación de TAC & & \\
$\quad$ Fracaso TAC y TEP crónico & $46(16)$ \\
$\quad$ Hemorragia & $19(6,6)$ \\
TEP masivo 0 TEP sumado a baja & $31(10,8)$ \\
reserva pulmonar & $6(2,1)$ \\
Profilaxis sin enfermedad tromboembólica & \\
Profilaxis con enfermedad tromboembólica, & \\
$\quad$ sumado a tratamiento anticoagulante & $33(11,5)$ \\
Embolia paradojal & 4 & $(1,4)$ \\
Flegmasia cerúlea dolens & 1 & $(0,4)$ \\
Filtro vena cava inferior previamente & $1(0,4)$ \\
$\quad$ mal posicionado & 1 &
\end{tabular}

tromboembólica, por presencia de trombo flotante en vena cava inferior, o territorio ilio-femoral en 27 pacientes. Filtros profilácticos en ausencia de enfermedad tromboembólica se indicaron sólo excepcionalmente en pacientes politraumatizados ( $\mathrm{n}=2$ ), o con factores de riesgo no modificables de enfermedad tromboembólica $(n=4)$.

Hubo cuatro casos de embolia arterial paradojal, que corresponde al paso de trombos a través de un foramen oval permeable, desde el territorio venoso al arterial. En un caso, la embolia afectó a las extremidades superiores e inferiores, en otro a la arteria mesentérica superior. Previa colocación del filtro, ambos pacientes se intervinieron en forma exitosa de su episodio embólico arterial. En los dos casos restantes la embolia afectó el territorio cerebrovascular.

Los filtros utilizados se describen en la Tabla 4. Se implantaron todos en forma permanente. El filtro Venatech ${ }^{\circledR}$ (Braun ${ }^{\circledR}$, Alemania), es el que

Tabla 3. C ausas de contraindicación o complicación del tratamiento anticoagulante en $\mathbf{1 6 0}$ pacientes

\begin{tabular}{|lrr|}
\hline Causa & $\mathrm{n}$ & $(\%)$ \\
\hline Perioperatorio & 68 & $(42,5)$ \\
Hemorragia intracraneana & 24 & $(15)$ \\
Hemorragia digestiva & 16 & $(10)$ \\
Hemoptisis & 5 & $(3,1)$ \\
Hemorragia intra o retroperitoneal & 4 & $(2,5)$ \\
Otras causas & 43 & $(26,9)$ \\
\hline
\end{tabular}


Tabla 4. Tipo de filtro utilizado en 287 pacientes

\begin{tabular}{|lrr|}
\hline Modelo & $\mathrm{n}$ & $\%$ \\
\hline Venatech $^{\circledR}$ (Braun, Alemania) & 101 & 35,2 \\
OptEase/TrapEase ${ }^{\circledR}$ (Cordis, EE.UU.) & 91 & 31,7 \\
Greenfield titanio $^{\circledR}$ (Boston Scientific, EE.UU.) & 67 & 23,3 \\
Günther-Tulip $^{\circledR}$ (Cook, EE.UU.) & 15 & 5,2 \\
Bird's Nest $^{\circledR}$ (Cook, EE.UU.) & 2 & 0,7 \\
\hline
\end{tabular}

hemos utilizado con mayor frecuencia. La selección del tipo de filtro dependió de la preferencia del tratante 0 disponibilidad al momento del implante, excepto por dos pacientes que recibieron filtros "Bird's Nest" por tener una vena cava cuyo diámetro excedía $28 \mathrm{~mm}$, límite máximo para garantizar que los filtros de diseño cónico se fijen a la pared de la vena y no se desplacen. La vía de acceso fue la vena yugular interna en 191 pacientes $(66,6 \%)$, la vena femoral común fue utilizada en 94 casos (32,8\%). El acceso subclavio fue excepcional $(0,6 \%)$.

En 24 pacientes $(8,4 \%)$ fue necesario implantar el filtro en posición suprarrenal, dada la presencia de trombo en la cava infrarrenal.

No hubo fracasos en la inserción, hematomas o infección del sitio de punción. Un paciente presentó un neumotórax en relación a la punción yugular, fue tratado con instalación transitoria de una pleurostomía, evolucionando sin complicaciones. No ha habido desarrollo de fístulas arteriovenosas clínicamente evidentes en el sitio de punción.

Tabla 5. C ausas de muerte en pacientes que fallecieron dentro de los 30 días posteriores a la instalación del filtro

\begin{tabular}{|lc|}
\hline Causa de muerte & $\mathrm{n}$ \\
\hline Respiratoria no embolia pulmonar & 6 \\
Embolia pulmonar & 3 \\
Sepsis & 3 \\
Cáncer & 3 \\
Hematoma mediastínico & 1 \\
Desconocido & 1 \\
\hline
\end{tabular}

La mortalidad a 30 días fue de 6,3\% (18 pacientes). Tres de ellos a consecuencia de la embolia pulmonar masiva original que motivó la inserción del filtro. Las otras causas más frecuentes fueron de origen respiratorio no secundarias a embolia pulmonar, sepsis y cáncer (Tabla 5). No hubo muertes relacionadas al procedimiento.

Se obtuvo un seguimiento promedio de 41,5 meses (rango: 0-144), éste fue completo en 246 pacientes $(85,7 \%)$ y parcial en 35 (12,2\%). Sólo 6 pacientes $(2,1 \%)$ no fuemon ubicables después del alta.

En el seguimiento a largo plazo, se han documentado 6 episodios de embolia pulmonar recurrente $(2,1 \%)$, en todos ellos el filtro había sido indicado por trombosis venosa profunda o embolia pulmonar con complicación o contraindicación del tratamiento anticoagulante. Tres de éstos fallecieron (1\%). En 22 pacientes $(7,7 \%)$, se demostró enfermedad trombótica sintomática recurrente, ya sea de extremidades inferiores o de vena cava, ninguno recibió el filtro como profilaxis en ausencia de enfermedad tromboembólica. No hemos observado otras complicaciones descritas en la literatura, como migración, fistulización u otra. En los casos de instalación suprarrenal del filtro, no hemos detectado complicaciones renales a largo plazo.

Durante el seguimiento 73 pacientes fallecieron después de 30 días (25,4\%). La causa más frecuente fue cáncer (22 casos, 30,1\%). En tres pacientes se desconoce la causa de muerte (Tabla 6). La sobrevida actuarial a 5 años fue de 64,7\% (Figura 1).

\section{DisCUSIÓN}

La trombosis venosa profunda es una patología frecuente en el ambiente hospitalario en general y 
Tabla 6. Causas de muerte en pacientes que fallecieron después de los 30 días posteriores a la instalación del filtro

\begin{tabular}{|lrr|}
\hline Causa de muerte & $\mathrm{n}$ & $\%$ \\
\hline Cáncer & 22 & 30,1 \\
Sepsis & 13 & 17,8 \\
Cardiovascular & 13 & 17,8 \\
Respiratoria no embolía pulmonar & 9 & 12,3 \\
Cerebrovascular & 5 & 6,8 \\
Embolia pulmonar & 3 & 4,1 \\
Otras & 8 & 11 \\
\hline
\end{tabular}

quirúrgico en particular, que puede llevar a complicaciones fatales precoces y discapacitantes tardías. Es sabido que su mejor control se logra con una adecuada profilaxis, pero una vez acaecida, deberán tomarse medidas para mejorar su pronóstico.

Homans, en 1944, inició la interrupción de la vena cava inferior como tratamiento de la enfermedad tromboembólica, con la ligadura simple de la vena cava. Con el fin de evitar la oclusión de la vena cava y sus secuelas, durante la década 195059 aparecieron diferentes modalidades de plicaturas quirúrgicas y aplicación de clips a la vena cava.

En 1967, Mobin-Uddin desarrolló un filtro con forma de paraguas, que podía insertarse desde la vena yugular interna denudada bajo anestesia local. Este filtro cayó en desuso debido a la alta tasa de oclusión de la vena cava y de migración del dispositivo, lo que corroboramos en nuestra experiencia inicial ${ }^{1}$.

En 1973, Greenfield desarrolló un filtro de forma cónica. Con este filtro existe extensa experiencia reportada ${ }^{2-5}$. Los primeros dispositivos requerían denudación de la vena yugular interna o femoral común. Durante la década de 1990-99, aparecieron filtros cuya inserción es posible por vía percutánea, sin detrimento de los resultados a corto y largo plazo ${ }^{2}$.

Basados en experiencias de otros grupos, menor perfil de inserción y resultados comparables in vitro, hemos incorporado otros filtros a nuestra práctica como alternativa al filtro de Greenfield ${ }^{6-8}$.

Al igual que otros ${ }^{3-5}$, usamos preferentemente la vía yugular interna por el riesgo teórico de desplazar trombos al utilizar la vía femoral. Hemos también preferido realizar este procedimiento en pabellón quirúrgico, con rigurosas medidas de asepsia y monitorización, para ofrecer las mejores condiciones de seguridad y la posibilidad de resolver una eventual complicación por el mismo equipo.

Nuestras indicaciones de interrupción de la vena cava inferior son similares a las reportadas por otros ${ }^{9,10}$. La más frecuente fue contraindicación del tratamiento anticoagulante o sus complicaciones.

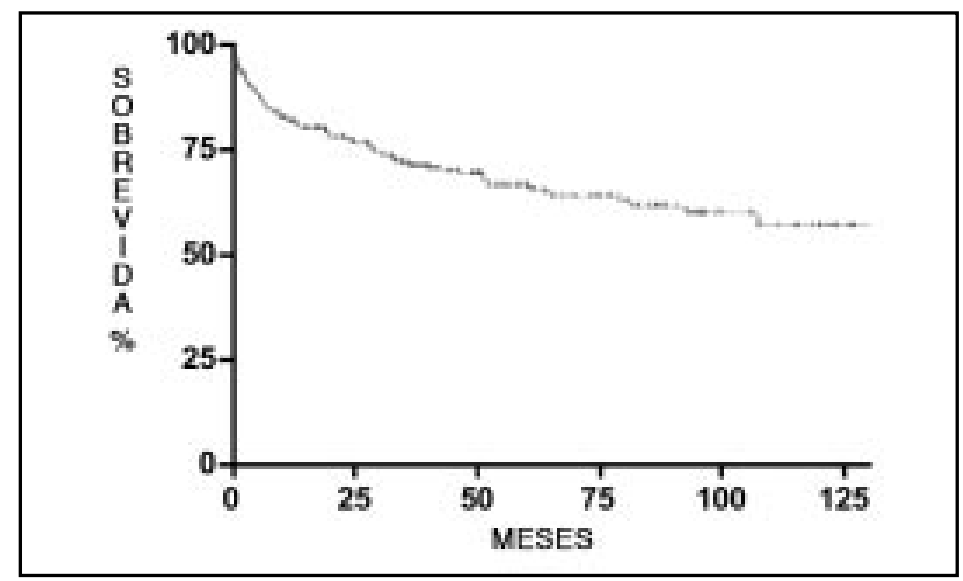

Figura 1. Gráfico de sobrevida actuarial de 287 pacientes sometidos a inserción de filtro de vena cava inferior. 
La indicación de filtros profilácticos, infrecuente en nuestra serie, se apoya en hechos conocidos, como la alta incidencia de embolia pulmonar pese a una anticoagulación efectiva (hasta 9\%), en pacientes con trombo flotante en el sector ilio femoral y vena cava ${ }^{11}$. Por otro lado, en pacientes hospitalizados por trauma grave, como fracturas de pelvis y huesos largos, se ha demostrado una disminución significativa de la incidencia de embolia pulmonar mediante la instalación de un filtro, comparado a medidas de profilaxis tradicionales. Se ha reportado incidencias entre $0 \%$ y $2 \%$ de embolia pulmonar en seguimiento entre $19 \mathrm{y}$ 42 meses promedio ${ }^{12-15}$ en este grupo de pacientes tratados con filtro. Dado el bajo volumen de pacientes politraumatizados en nuestro centro, ésta fue una indicación infrecuente en esta serie.

La mortalidad derivada del procedimiento ha sido cercana a cero en múltiples series, como en la presente ${ }^{10}$. Sin embargo, la mortalidad a 30 días puede ser elevada como es el caso de este reporte y otros, encontrando en la literatura tasas de hasta $17 \%$ de mortalidad a 30 días $^{3-5,9,10}$. Esto debido a la enfermedad de base o sus complicaciones, especialmente sepsis y falla orgánica múltiple.

En el largo plazo se ha descrito una incidencia de embolia pulmonar recurrente entre $0 \%$ y $5,6 \% 3$ $5,9,10,13-16$. Nosotros observamos una incidencia de 2,1\% de embolia pulmonar posterior a la inserción del filtro. A su vez, la oclusión de la vena cava inferior es también de baja incidencia, reportándose entre 0,95\% y 5\%3-5,10,13,14.

Las complicaciones derivadas del filtro, si bien están comunicadas en la literatura, son extremadamente poco frecuentes. Han sido descritos casos de falla del material17,18, migración del filtro dentro del aparato circulatorio o su fistulización a órganos vecinos requiriendo ocasionalmente tratamiento quirúrgico o endovascular para su resolución ${ }^{3-5,19-26}$; la infección del filtro es un evento excepcional ${ }^{27}$. Es necesario considerar estas complicaciones en pacientes que posean un filtro en la vena cava y que se presenten con sintomatología poco habitual.

La instalación suprarrenal del filtro, frecuente en nuestra experiencia $(8,4 \%)$ es comparable a la incidencia de otras series más numerosas. No se ha demostrado morbilidad desde el punto de vista de función renal en nuestra experiencia y sólo se ha reportado como una rareza ${ }^{10,28-30}$.
En nuestros pacientes no se realizó control ultrasonográfico del sitio de punción, por lo que se desconoce la tasa de trombosis local postprocedimiento. Consideramos que el conocimiento de dicho dato no llevaría necesariamente a un cambio de conducta, por lo que no es clínicamente relevante. Tampoco se realizó seguimiento con imágenes del filtro y de la vena cava inferior en todos los pacientes, sino sólo a aquellos que se presentaron con sintomatología, por lo que nuestra incidencia de trombosis venosa recurrente 0 trombosis de vena cava puede estar subvalorada.

El estudio PREPIC ${ }^{31,32}$ sugiere que la presencia de un filtro de vena cava inferior reduce la incidencia de embolia pulmonar, sin afectar la mortalidad y determina un aumento en la incidencia de trombosis venosa profunda, reportando a 8 años de seguimiento, una tasa de $27,5 \%$ en pacientes que no recibieron un filtro, versus $35,7 \%$ en aquellos que sí lo recibieron. Lamentablemente, la mayoría de los pacientes que se incluyeron para la distribución aleatoria en este estudio, no tenían indicación clásica y universalmente aceptada de instalación de un filtro y además, se utilizó con frecuencia el filtro "Bird's Nest", que provoca una mayor incidencia de trombosis. Este es el único estudio prospectivo y aleatorio acerca de la utilidad de este procedimiento, pero adolece de estos importantes problemas metodológicos que invalidan, al menos en parte, sus conclusiones. En pacientes tratados médicamente por trombosis venosa profunda y que no han recibido un filtro de vena cava inferior se reportan incidencias entre $13 \%$ y $24,6 \%$ de trombosis venosa recurrente a 5 años de seguimiento ${ }^{33,34}$, del todo similares a las que se reportan en grupos de pacientes que poseen un filtro 10,13,14,32, como lo descrito en el presente trabajo. Dado esto, no resulta evidente que la presencia de un filtro cónico en la vena cava inferior constituya necesariamente un factor de riesgo para trombosis.

Basados en la existencia de pacientes que poseen un alto riesgo de eventos tromboembólicos o contraindicación de anticoagulación transitorios y en un eventual aumento de riesgo de trombosis venosa profunda derivada del filtro, han surgido como solución los filtros removibles de utilización transitoria. Diferentes grupos han reportado tasas de retiro del filtro que fluctúan entre $51 \%$ y $91 \% 8,35,36$. Las razones para no retirarlo en 
todos los pacientes es la presencia de trombos en el filtro o persistencia de la contraindicación de anticoagulación o del riesgo que lo indicó inicialmente. Nosotros no hemos aplicado esta técnica, ya que la indicación del retiro estaría basada en una alta incidencia de complicaciones trombóticas o de otro tipo en el largo plazo, lo que no tiene un claro fundamento ni en la literatura ni en

\section{REFERENCIAS}

1. Mertens R, Valdés F, Krämer A, Vergara J. Interrupción de vena cava inferior mediante filtros: indicaciones y resultados en 111 pacientes. Rev Méd Chile 1998; 126: 655-60.

2. Greenfield L, Proctor M, Cho K, Cutler B, Ferris E, McFARLAND D ET AL. Extended evaluation of the titanium Greenfield caval filter. J Vasc Surg 1994; 20: 458-65.

3. GREENFIELD L. Current indications for and results of Greenfield filter placement. J Vasc Surg 1984; 1: 502-4.

4. Greenfieid L, Michna B. Twelve-year clinical experience with the Greenfield vena caval filter. Surgery 1988; 104: 706-12.

5. Greenfield L, Peyton R, Crute S, Barnes R. Greenfield vena caval filter experience. Arch Surg 1981; 116: 1451-6.

6. Hammer F, Rousseau H, Joffre F, Sentenac B, TranVAN T, BARTHELEMY RP. In vitro evaluation of vena cava filters. JVIR 1994; 5: 869-76.

7. Xian Z, Sumit R, Hosaka J, Kvernebo K, Laerum F. Multiple emboli and filter function: An in vitro comparison of three vena cava filters. JVIR 1995; 6: 887-93.

8. Hoff W, Hoey B, Wainwright G, Reed J, Ball D, RINGold M ET AL. Early experience with retrievable inferior vena cava filters in high-risk trauma patients. J Am Coll Surg 2004; 199: 869-74.

9. Wojtowyez MM, Stoenr T, CRummy AB, Mcdermott JC, Sproat IA. The Bird's Nest inferior vena cava filter: a review of a single-center experience. J Vasc Interv Radiol 1997; 8: 171-9.

10. Athanasoulis C, Kaufman J, Halpern E, Walkman A, SeLLER S, FAN C. Inferior vena caval filters: review of a 26-year single center clinical experience. Radiology 2000; 216: 54-66. nuestra experiencia, agregando mayor costo y eventual morbilidad al manejo de la enfermedad tromboembólica.

Basados en nuestra experiencia y en la comunicada por otros, concluimos que el implante de un filtro de vena cava inferior es una medida de bajo riesgo inmediato y en el largo plazo y de alta utilidad en la profilaxis de embolia pulmonar.

11. Norris C, Greenfield L, Herrmann J. Free-floating iliofemoral thrombus. A risk of pulmonary embolism. Arch Surg 1985; 120: 806-8.

12. Khansarinia S, Dennis J, Veldenz $H$, Butcher J, HaRTLAND L. Prophylactic Greenfield filter placement in selected high-risk trauma patients. J Vasc Surg 1995; 22: 231-6.

13. GREenField L Prophylactic vena caval filtres in trauma: the rest of the story. J Vasc Surg 32: 490-7.

14. Wojcik R, Cipole M, Fearen I, Jaffe J, Newcomb J, PASQUALE M. Long-term follow -up of trauma patients with a vena cava filter. J Trauma 2000; 49: 839-43.

15. Langan E, Muler R, Casey W, Carsten C, Graham R, TAYLOR S. Prophylactic inferior vena cava filters in trauma patients at high risk: follow-up examination and risk benefit assessment. J Vasc Surg 1999; 30: 484-8.

16. GREENField L, PRoctor M. Recurrent thromboembolism in patients with vena cava filters. J Vasc Surg 2001; 33: 510-4.

17. Dabbagh A, Chakfé N, Kretz JG, Demri B, Nicolni P, FuENTES $C$ ET AL. Late complication of a Greenfield filter associating caudal migration and perforation of the abdominal aorta by a ruptured strut. J Vasc Surg 1995; 22: 182-7.

18. Plaus W, Hermann G. Structural failure of a Greenfield filter. Surgery 1988; 103: 662-4.

19. Kim D, Porter D, Seigel J, Simon M. Perforation of the inferior vena cava with aortic and vertebral penetration by a suprarenal Greenfield filter. Radiology 1989; 172: 721-3.

20. Kurgan A, Nunneiee J, Auer A. Penetration of the wall of an abdominal aortic aneurysm by a Greenfield filter prong: a late complication. J Vasc Surg 1993; 18: 303-6.

21. Kupferschmid J, Dickson C, Towndsend R, Diamond DL. Small bowel obstruction from an extruded 
Greenfield filter: an unusual late complication. J Vasc Surg 1992; 16: 113-5.

22. Mrtcheш WB, BonN J. Percutaneous retrieval of a Greenfield filter after migration to the left pulmonary artery. J Vasc Interv Radiol 2005; 16: 1013-7.

23. IzUTANi H, Lalude O, Gius I, Biblo L. Migration of an inferior vena cava filter to the right ventricle and literature review. Can J Cardiol 2004; 20: 233-5.

24. Stacey C, Manhire A, Rose D, Bishop M. Bird's nest filter causing symptomatic hydronephrosis following transmural penetration of the inferior vena cava. Cardiovasc Intervent Radiol 2004; 27: 61-3.

25. Raghavan S, AKhtar A, Bastani B. Migration of inferior vena cava filter into renal hilium. Nephron 2002; 91: 333-5.

26. Shaer J, Epstein N. An unusual cause of low back pain? A case report. Spine 1998; 23: 1349-50.

27. Shimizu M, Tatsumi K, Matsukawa R, Shima T, Miwa Y. Retrievable Günther Tulip filter complicated by sepsis and retroperitoneal hemorrhage: successful management by filter retrieval. Intern Med 2005; 44: 593-7.

28. Greenfield L, Cho K, Proctor M, Sobel M, Shah S, Wingo J. Late results of suprarenal Greenfield vena cava filter placement. Arch Surg 1992; 127: 969-73.

29. GreEnfield L, PRoctor M. Suprarenal filter placement. J Vasc Surg 1998; 28: 432-8.

30. Marcy P, Magne N, Frenay M, Bruneton J. Renal failure secondary to thrombotic complications of suprarenal inferior vena cava filter in cancer patients. Cardiovasc Intervent Radiol 2001; 24: 257-9.

31. Decousus H, Leizorovicz A, Parent F, Page Y, Tardy B, GirARd P ET AL. A clinical trial of vena caval filters in the prevention of pulmonary embolism in patients with proximal deep-vein thrombosis. N Eng J Med 1998; 228: 409-15.

32. The PREPIC Study Group. Eight-year follow-up of patients with permanent vena cava filters in the prevention of pulmonary embolism. Circulation 2005; 112: 416-22.

33. Beyth R, Cohen A, Landefeid C. Long-term outcomes of deep-vein thrombosis. Arch Intern Med 1995; 155: 1031-7.

34. Prandoni P, Lensing AW, Cogo A, Cuppini S, Vilalta S, CaRTA M ET AL. The long-term clinical course of acute deep venous thrombosis. Ann Intern Med 1996; 125: 1-7.

35. Milward S, Oliva V, Bell S, Valenti D, Rasuli $P$, Asch M et aL. Günther Tulip Retrievable Vena Cava Filter: results from the Registry of the Canadian Interventional Radiology Association. J Vasc Interv Radiol 2001; 12: 1053-8.

36. Grande W, Trerotola S, Reiwy P, Ciark T, Soulen M, Patel A ET AL. Experience with the recovery filter as a retrievable inferior vena cava filter. J Vasc Interv Radiol 2005; 16: 1189-93.

37. The Participants in the Vena Caval Filter Consensus CONFERENCE. Recommended reporting standards for vena caval filter placement and patient follow-up. J Interven Radiol 2003; 14: S427-S432. 